\title{
A Regularized Deep Learning Approach for Image De-Blurring
}

\author{
Francesco Lo Conti \\ Advanced Global Solution AGS S.p.A \\ Pero, Italy \\ francesco.loconti@ags-it.com
}

\author{
Gabriele Minucci \\ Advanced Global Solution AGS S.p.A \\ Pero, Italy \\ gabriele.minucci@ags-it.com
}

\author{
Naser Derakhshan \\ Advanced Global Solution AGS S.p.A \\ Pero, Italy \\ naser.derakhshan@ags-it.com
}

\begin{abstract}
In this paper, a novel convolutional neural network model for blind deconvolution of images is proposed. The structure of the model is based on two sub models devoted, respectively, to deblurring and denoising of an input image. The model has been designed to restore a picture affected by different kinds of noise. The main innovation is the introduction of a regularization term in the training cost function, based on a blurred/non-blurred classification tool. Results show interesting features of the model, particularly regarding the robustness of results. The comparison with other state-of-the-art models confirms the value of the model proposed in this study.
\end{abstract}

\section{CCS CONCEPTS}

- Computing methodologies $\rightarrow$ Neural networks; Regularization; Supervised learning;

\section{KEYWORDS}

Machine Learning, Deep Learning, Convolutional Neural Network, Blind Image De-Blurring, Image Restoration

\section{ACM Reference Format:}

Francesco Lo Conti, Gabriele Minucci, and Naser Derakhshan. 2017. A Regularized Deep Learning Approach for Image De-Blurring. In IML '17: International Conference on Internet of Things and Machine Learning, October 17-18, 2017, Liverpool, United Kingdom. ACM, New York, NY, USA, 5 pages. https://doi.org/10.1145/3109761.3158383

\section{INTRODUCTION}

In the last decade, we have faced a vast amount of digital images in different application domains, from personal mobile devices to medical equipment and from sports cameras to telescopes. These pictures can be blurry for some reason, such as camera motion, image compression, and being out-of-focus. The availability of these large amounts of images stimulates the need for tools that can enhance their quality. The objective of this work is to restore a blurred image without any prior information about the picture called blind deconvolution ${ }^{1}$. Since in a blind approach information about the blurring process is not provided, it can be demonstrated that the problem is ill-posed[9] from an analytical point of view.

\footnotetext{
${ }^{1}$ The inversion of blurring process is called image deconvolution. In this paper, decon volution and de-blurring are used interchangeably.

Permission to make digital or hard copies of part or all of this work for personal or classroom use is granted without fee provided that copies are not made or distributed for profit or commercial advantage and that copies bear this notice and the full citation on the first page. Copyrights for third-party components of this work must be honored. For all other uses, contact the owner/author(s).

IML '17, October 17-18, 2017, Liverpool, United Kingdom

(c) 2017 Copyright held by the owner/author(s).

ACM ISBN 978-1-4503-5243-7/17/10.

https://doi.org/10.1145/3109761.3158383
}

In this study, a novel convolutional neural network for blind image deconvolution is proposed. Although this is a long-standing problem and similar solutions have already been proposed $[14,17$, $21,21,23]$ there is room for improvement over the best existing methods. The contribution of this work is related to the introduction of a regularization term in the cost function adopted for the training that facilitates the convergence of the model toward not-blurred images. The proposed solution can deblur a blurry image, and at the same time, it can keep the quality of input images which are not blurred at all. Moreover, the final results of the network on blurry images show improvements in the quality of the output compared to the similar available solutions in the literature.

The rest of the paper is organized as follows. Section 2 introduces the state of the art. In Section 3 an introduction to algorithms for blind deconvolution is provided, while Section 4 describes the proposed model for image deconvolution. Finally, evaluation of the proposed model is provided in Section 5.

\section{RELATED WORK}

Over the last few years, many works have been done on deblurring digital images. Most of these studies have been developed as optimization problems and referred to either a total variation (TV) approach $[1,3,4]$ or a maximum a priori probability (MAP) approach $[8,12,13]$. Assessments of these models often display poor results concerning the variability of blurring filters and noise of images. Furthermore, such approaches rely heavily on certain assumptions about the properties of the original de-convoluted image. However, given the enormous dimensionality of the space of the original image, posing such assumptions yields an oversimplified modeling of the problem.

Sun et al.[17] proposed a model for finding the blur kernel within an image. This model does not provide a tool for recovering the deblurred image while performing the denoising of the deconvoluted image.

Recently, deep learning approaches have been proposed for image restoration problems. Deep learning algorithms build an inner generative model based on the data. This model is typically much more complex and close to the real nature of the data, given that training samples describe the space of the data correctly. Therefore the greatest advantage of such approaches lies in their ability to use a better generative model of the original image to drive the restoration of the convoluted picture. Xu et al. [20] used an auto-encoder model for image denoising and in-painting tasks. Although this model can restore images for a wide range of modifications, it does not accomplish image deblurring.

Many works have been done on non-blind image deconvolution using a machine learning approach[14, 16, 24]. Although they present the proper results about image deblurring, the non-blind approach requires some information about the image a priori and 
cannot be used in many application domains, for which no information is available about the image a priori. For instance, Schuler et al. [14] proposed a machine learning approach for non-blind image deconvolution. They adopted an MLP (multi-layer perceptron) network for their model. Their evaluation results showed state-of-the-art results when trained on a specific kernel, but the performance of their model decreases significantly when facing multiple blur types. Moreover, as stated before, this approach requires some information about the image a priori.

Blind deblurring has been a long-studied task (e.g. [2, 6, 7, 10, 11, 15, 19, 21]). Specifically, Chrysos et al.[6] presented a blind deblurring method for human faces. However, their solution is domain-specific and cannot be applied to many application domains. The authors of [19], and [10] introduce specific statistical tools in an optimization framework. Even though the adoption of these tools can enhance the performance of more general analytical methods, they can lead to unsatisfactory results where the hypotheses at their basis are not respected. There are other similar works $[7,11]$ that adopt deep learning solutions for non-blind deconvolution but within a restricted application domain, such as microscopic imagery and motion blurred images. Another deep learning approach is proposed by Schuler et al. [15]. However, it fails for large size kernels.

Finally, Xu et al.[21] proposed a DCNN (deep convolutional neural network) for recovering blurry and noisy images in a blind deconvolution framework. This model appears as one of the most important proposals for our image deblurring model, using deep learning methods. However, their model will fail if the image is not blurry. In this case, the output image would have worse quality than the input image. In this paper, we propose a model that solves this very last limitation. Moreover, our experimental results show that our approach outperforms the state-of-the-art methods on blind image deconvolution.

\section{BLIND DECONVOLUTION ALGORITHMS}

The image deconvolution theoretical framework is given by the inverse problem of recovering the base version $x$ of an input image $y$ obtained as a convolution of $x$ with a filter $k$ (often indicated as PSF, point spread function) and considering a random noise $n$ :

$$
y=x * k+n
$$

Random noise could be attributed to image saturation and compression artifacts. Image deconvolution problems arise when, given the blurred image $y$, the original picture $x$ has to be retrieved. If the PSF at the basis of convolution is known, the deconvolution is said to be non-blind. Otherwise, the problem is indicated as blind deconvolution.

Main developments of both problems are linked to the application of the deconvolution theorem that allows considering the convolution as an element-wise product of Fourier transformed variables. In this way, it is possible to approximate the deconvolution task introducing a pseudo-inverse kernel $k^{*}$. The Fourier transform of such a pseudo-inverse kernel can be computed as the reciprocal of the Fourier transform of $k$ :

$$
F(y)=F(x) \cdot F(k)
$$

$$
\begin{gathered}
F(x)=\frac{F(y)}{F(k)}=F\left(k^{*}\right) \cdot F(y) \\
x=F^{-1}\left(k^{*}\right) * y .
\end{gathered}
$$

In Equations 2, 3, and $4 F$ and $F^{-1}$ denote respectively the Fourier transform and the inverse Fourier transform. Since the noise term has been neglected, and to avoid division-by-zero in the frequency domain, a regularization term is usually introduced. The Wiener deconvolution [18] corresponds to the implementation of such a regularization and can be expressed as follows:

$$
x=F^{-1}\left(\frac{1}{F(k)}\left(\frac{|F(k)|^{2}}{|F(k)|^{2}+\frac{1}{S N R}}\right)\right) * y=k^{i n v} * y
$$

where $S N R$ is the Signal to Noise Ratio and $k^{i n v}$ synthesizes the terms relative to the pseudo-inverse kernel.

Problems related to non-blind deconvolution mainly deal with the quality and the computational efficiency methods to perform the retrieving of $x$. Moreover, such methods do not always succeed to be robust concerning possible noise effects in the output image [21].

Blind deconvolution methods constitute an analytical ill-posed problem, since not a single $x, k$ couple exists for a given blurred image $y$. To deal with this problem, a further constraint on the problem can be introduced, considering characteristics of natural, i.e., not blurred images. In particular, it has been pointed out that gradient distribution of such images is usually the sparsest among the possible retrievable images $[5,8,13]$.

\section{PROPOSED MODEL}

Our model is inspired by the technique introduced in [21]. Their result is achieved using a neural network with convolutional layers constituted by filters with large sizes that are supposed to effectively reproduce the suited inverse filters for an input blurred image. Because of the nature and the size of these layers, a proper initialization is needed to optimize the representation of the inverse filters. Indeed, the initialization of deblurring layers is carried out, defining an inversion process of a set of blurring filters that are supposed to represent the range of effects observed in input images. The inversion could be performed retrieving $k^{i n v}$ as described in Equation 5. However, the authors used the inversion technique described in [22] that is claimed to be numerically more robust. Moreover, spatial filters are decomposed into the combination of two 1D filters. Finally, the denoising sub model is constituted by a list of convolutional layers with small size filters that exploit the depth information collected among themselves.

Drawbacks of this model can be associated with the initialization of well-suited deblurring filters and the training of a massive neural network architecture. The model provided in this study proposes a modified version of this approach, where proper solutions for these issues are introduced.

In this section, first, the model used is described, along with the selection of the training cost function. Then the data retrieving activity and the training strategy are outlined.

Similar to work in [21], we also developed a DCNN model for deblurring and denoising images with a blind approach. Several 
differences have been introduced, related to the architecture, the cost function, and the training of the model.

To be able to visualize and interpret deblurring filters, single 2D convolutional layers have been used instead of two 1D layers in the deblurring sub model. Another difference between our model and the one proposed by [21] regards the depth of convolutional layers for the denoising sub model. Design experiments showed that this size was much too expensive concerning the need of restoring images mainly affected by blurring effects. Therefore, to reduce the complexity of the network, the depth of denoising layers has been decreased.

Finally, the training has been performed using a regularized cost function. The regularization term is based on the output of a classification model designed and trained for distinguishing between blurred and non-blurred images.

Networks for classification and deblurring are illustrated in Figure 1.

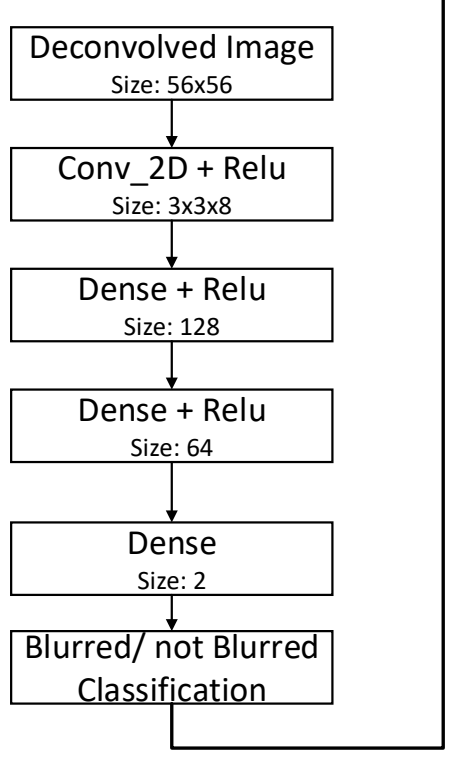

(a)

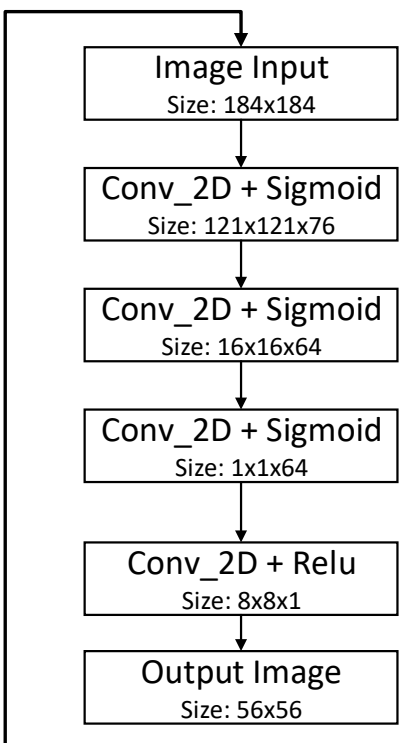

(b)
Figure 1: Models proposed in this study. (a) Classification model. (b) De-blurring and de-noising model.

The output of the classification model is provided on a continuous scale, with lower values associated with non-blurred images and higher values related to blurred images. Such an evaluation is carried out using the assessment given by the classification model, weighted with a probability of reliability. Motivations behind the need for the introduction of such a reliability measure arise from the observation that often images do not contain enough information for a reliable classification. Therefore, to account for this effect, a coefficient that measures the information available for the deblurring has been designed. Such a coefficient is given by the following relationship:

$$
s_{i}=\phi\left(\beta \cdot \max \left(\sigma\left(p_{i}\right)\right)+\gamma\right)
$$

where $s_{i}$ is the corrective coefficient for the $i$-th image, $\phi$ is the logistic function, $\sigma\left(p_{i}\right)$ is the mean standard deviation of image patches in the $i$ image, $\beta$ and $\gamma$ are specific coefficients. These coefficients have been calibrated evaluating thresholds values of $\sigma\left(p_{i}\right)$ corresponding to non-informative images and maximum valuable informative pictures. The final expressions for the computation of the cost function is reported in Equation 7:

$$
\cos t=\frac{1}{N} \sum_{i=1}^{N}\left(y_{i}-\hat{y}_{i}\right)^{2}+\alpha \sum_{i=1}^{N}\left(s_{i} \cdot b_{i}\right)
$$

where $y_{i}$ is the $i$-th image provided for the cost computation, $N$ is the number of such images, $\hat{y}_{i}$ is the target value, $\alpha$ is a regularization coefficient, $b_{i}$ is the result of the classification, and $s_{i}$ is the weight relative to the level of image information available for the deblurring.

The final cost function designed in this way is supposed to penalize output images that are evaluated as still blurred, and therefore, to lead the training toward a picture classified as non-blurred. The effectiveness of these assumptions has been practically evaluated during the calibration of the $\alpha$ coefficient. Such a process has been pursued, repeating the training for several values of the alpha coefficient and evaluating performances on a benchmark of images. Results are reported in Section 5.

\subsection{Training Insights}

To build the dataset for the training of the models, a collection of large size and possibly good quality (i.e., without noise) wallpaper from the web has been downloaded. A procedure to add a Gaussian blur effect and several types of noise has been designed and applied to each image. Specifically, this procedure includes the application of a Gaussian blur, a jpeg compression, a saturation effect, and random noise. Parameters of each effect are randomly selected from a specific range to reproduce their variability.

The classification model has been trained randomly, providing, as input, original or noisy images, respectively, with a target value equal to zero and one.

The complexity of the deconvolution network and the high number of parameters to be calibrated implies some difficulties for its training. To tackle this issue, a warm-up has been performed with a short training, using a subset of the training dataset. After this warm-up, the training was switched to a standard stochastic training.

The training relative to the deconvolution network demonstrated to be highly computationally demanding. To carry out both the starting experiments and to train the network, a server called HPC $90^{2}$ assembled by the AGS S.p. A $^{3}$ with an array of eight GPUs has been prepared, along with a library to implement a data-parallelism framework. Specifically, eight Nvidia GeForce GTX 1080 Ti have been used to split the training batch size equally. The hardware description is stated in Table 1.

\section{EVALUATION}

The trained classification model can correctly classify $\backsim 80 \%$ of the test images as blurred/non-blurred. It has been verified that

\footnotetext{
${ }^{2}$ https://www.ags-it.com/hpc.html

${ }^{3}$ www.ags-it.com
} 
Table 1: Machine hardware specification used for the training.

\begin{tabular}{|c|c|}
\hline Feature & Specification \\
\hline Model & HPC 90 (Assembled by AGS S.p.A) \\
\hline CPU & $\begin{array}{c}\text { Intel(R) Xeon(R) CPU E5-2630 v4 @ 2.20GHz } \\
\text { (2 sockets, 10 Cores, 40 virtual CPUs) }\end{array}$ \\
\hline RAM & 128 GB \\
\hline GPU & Nvidia GeForce GTX 1080 Ti - 8 Units \\
\hline
\end{tabular}

images not correctly classified have low information. Therefore, these pictures do not influence the cost function, as the value of $s_{i}$ penalizes such images.

The evaluation of the deconvolution model is performed on a collection of benchmark images. The Gaussian blurring technique that has been applied to this collection is the same as the one we applied to the training images.

A quantitative measure of image deblurring achieved by the model is obtained computing the Peak Signal to Noise Ratio (PSNR) between the output image and the original version. PSNR provides higher values proportionally to the goodness of restoration, as it implies the computation of the MSE (Mean Square Error) between the images:

$$
P S N R=20 \cdot \log _{10}\left(\frac{\text { peakvalue }}{\sqrt{M S E}}\right)[d B]
$$

with peakvalue being the maximum theoretical value for input images. Both blurred and deblurred images are compared with the original version using the PSNR (Table 2). De-blurred images systematically display an enhancement regarding PSNR value.

A first analysis has been performed for the calibration of the regularization coefficient $\alpha$. The training has been repeated for several values of $\alpha$, along with the evaluation of the PSNR for the benchmark images (Figure 2). Results show that the best value is achieved by $\alpha=0.2$. This result demonstrates that the introduction of the regularization term improves the performance of the training, as the results obtained for $\alpha$ greater than zero outperform those for $\alpha$ equal or close to zero.

Using the calibrated model, an evaluation of the model has been applied to benchmark images (Figure 3).

The model proposed has been compared with two other models available in the literature, i.e., the model proposed by Xu et al. [21] and the algorithm proposed by Krishnan et al. [12] that respectively represent a reference for deep learning and optimization approaches. This comparison has been performed on blurred images with a more relevant disturbance level that is more closely related to real cases. Results, in terms of PSNR, are reported in Table 3. As can be observed, even though values often differ very slightly and a clear ranking of the models cannot be depicted, the model proposed in this study outperforms other models for several images and it can retrieve a de-blurred image with PSNR value greater than that of the input image.

\section{CONCLUSIONS AND FUTURE WORK}

In this paper, a new blind image deconvolution model has been proposed. The model is given by a convolutional neural network

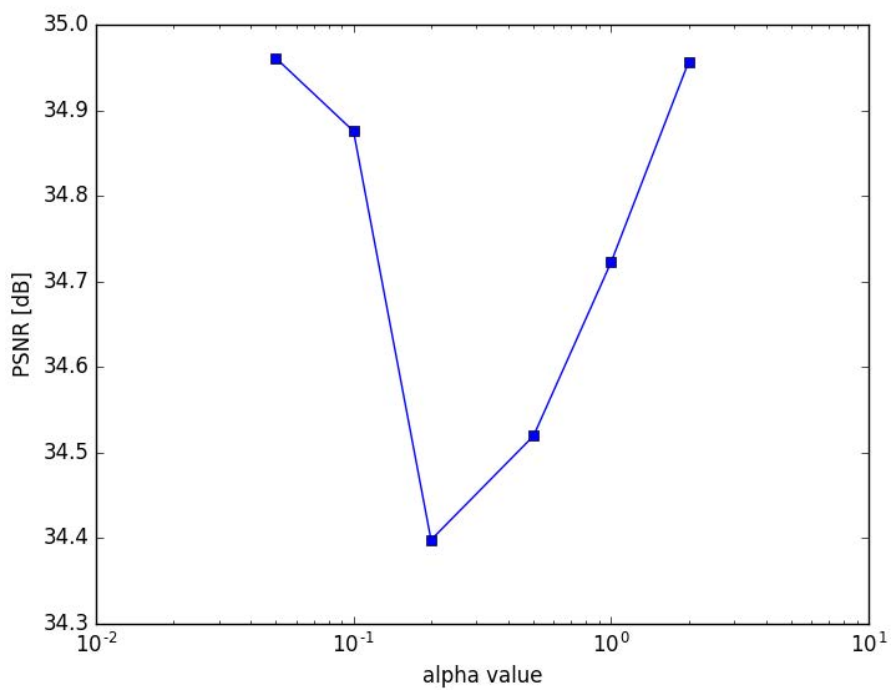

Figure 2: Values of PSNR [dB] along with alpha coefficient values.

Table 2: PSNR [dB] of results.

\begin{tabular}{|c|c|c|}
\hline Image & PSNR original-blurred & PSNR original-deblurred \\
\hline a & 27.525 & 30.943 \\
\hline b & 28.286 & 30.019 \\
\hline c & 29.072 & 32.233 \\
\hline d & 28.906 & 30.157 \\
\hline e & 25.281 & 26.913 \\
\hline f & 30.082 & 33.437 \\
\hline
\end{tabular}

Table 3: PSNR [dB] obtained comparing results from our model, Xu et al. [21], and Krishnan et al. [12] for images with high level blurring.

\begin{tabular}{|c|c|c|c|}
\hline Image & PSNR our method & PSNR [21] & PSNR [12] \\
\hline a & 17.975 & 16.249 & 17.367 \\
\hline b & 20.930 & 20.787 & 19.518 \\
\hline c & 19.360 & 17.410 & 18.685 \\
\hline d & 19.232 & 18.970 & 17.572 \\
\hline e & 17.039 & 16.276 & 16.070 \\
\hline f & 15.825 & 15.092 & 17.281 \\
\hline
\end{tabular}

trained with the introduction of a regularization term in the cost function that boosts the identification of de-blurred images. Results confirm that the output retrieved by the model for input blurred images appears de-blurred and a PSNR measure confirms the improvement of pictures.

\section{REFERENCES}

[1] S. D. Babacan, R. Molina, and A. K. Katsaggelos. 2009. Variational Bayesian blind deconvolution using a total variation prior. IEEE Transactions on Image Processing 18, 1 (2009), 12-26.

[2] S. A. Bigdeli and M. Zwicker. 2017. Image Restoration using Autoencoding Priors. CoRR abs/1703.09964 (March 2017). 
(a)
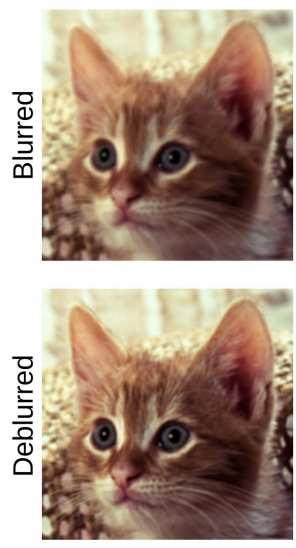

(d)
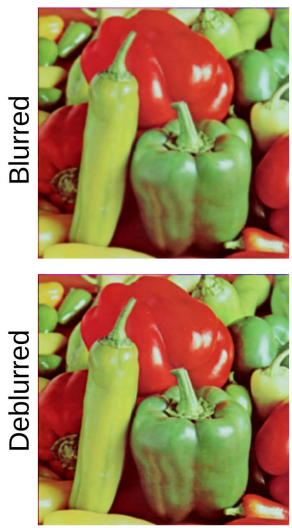

(b)
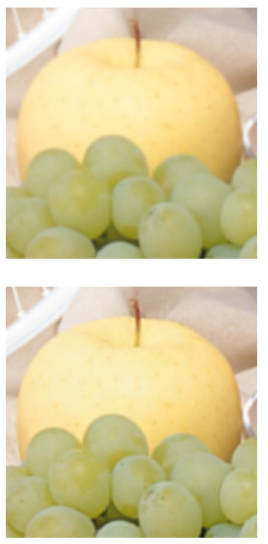

(e)
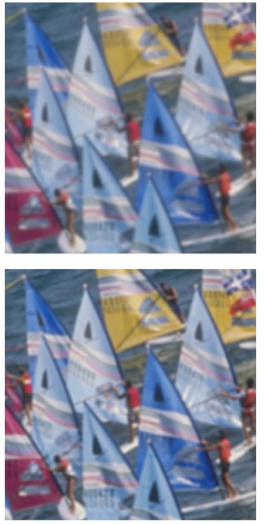

(c)
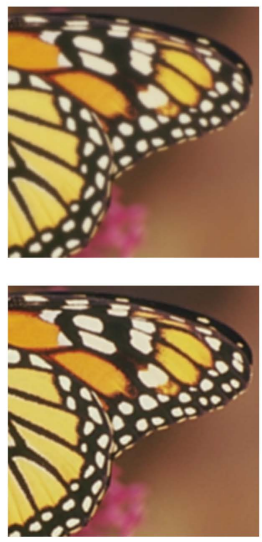

(f)
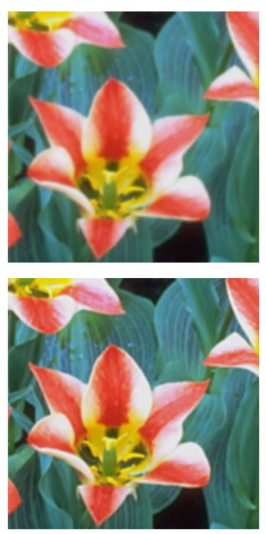

Figure 3: Results of deblurring in test images.

[3] P. Blomgren, T. F. Chan, P. Mulet, and C. K. Wong. 1997. Total variation image restoration: numerical methods and extensions. In International Conference on Image Processing, Vol. 3. 384-387.

[4] T. F. Chan and C. K. Wong. 1998. Total variation blind deconvolution. IEEE transactions on Image Processing 7, 3 (1998), 370-375.

[5] S. Cho and S. Lee. 2009. Fast motion deblurring. In ACM Transactions on Graphics (TOG), Vol. 28. 145

[6] G. G. Chrysos and S. Zafeiriou. 2017. Deep Face Deblurring. In IEEE Conference on Computer Vision and Pattern Recognition Workshops (CVPRW). 2015-2024.

[7] J. Cronje. 2015. Deep convolutional neural networks for dense non-uniform motion deblurring. In International Conference on Image and Vision Computing New Zealand (IVCNZ). 1-5.

[8] R. Fergus, B. Singh, A. Hertzmann, S. T. Roweis, and W. T. Freeman. 2006. Removing camera shake from a single photograph. In ACM Transactions on Graphics (TOG), Vol. 25. 787-794.

[9] J. Hadamard. 1902. Sur les problèmes aux dérivés partielles et leur signification physique. Princeton University Bulletin 13 (1902), 49-52.

[10] I. S. Jeon, D. Kang, and S. I. Yoo. 2017. Blind image deconvolution using Student's$\mathrm{t}$ prior with overlapping group sparsity. In IEEE International Conference on Acoustics, Speech and Signal Processing (ICASSP). 1817-1821.

[11] T. Kenig, Z. Kam, and A. Feuer. 2010. Blind Image Deconvolution Using Machine Learning for Three-Dimensional Microscopy. IEEE Transactions on Pattern Analysis and Machine Intelligence 32, 12 (Dec 2010), 2191-2204.

[12] D. Krishnan, T. Tay, and R. Fergus. 2011. Blind deconvolution using a normalized sparsity measure. In IEEE Conference on Computer Vision and Pattern Recognition (CVPR). 233-240.

[13] A. Levin, Y. Weiss, F. Durand, and W. T. Freeman. 2011. Efficient marginal likelihood optimization in blind deconvolution. In IEEE Conference on Computer Vision and Pattern Recognition (CVPR). 2657-2664.

[14] C. J. Schuler, H. C. Burger, S. Harmeling, and B. Schölkopf. 2013. A Machine Learning Approach for Non-blind Image Deconvolution. In IEEE Conference on Computer Vision and Pattern Recognition. 1067-1074.

[15] C. J. Schuler, M. Hirsch, S. Harmeling, and B. Schölkopf. 2016. Learning to Deblur. IEEE Transactions on Pattern Analysis and Machine Intelligence 38, 7 (July 2016), 1439-1451.

[16] H. Son and S. Lee. 2017. Fast non-blind deconvolution via regularized residual networks with long/short skip-connections. In IEEE International Conference on Computational Photography (ICCP). 1-10.

[17] J. Sun, W. Cao, J. Xu, and J. Ponce. 2015. Learning a convolutional neural network for non-uniform motion blur removal. In Proceedings of the IEEE Conference on Computer Vision and Pattern Recognition. 769-777.

[18] N. Wiener. 1949. Extrapolation, interpolation, and smoothing of stationary time series. Vol. 7. MIT press Cambridge, MA.

[19] D. Wipf and H. Zhang. 2014. Revisiting Bayesian Blind Deconvolution. 7. Mach. Learn. Res. 15, 1 (Jan 2014), 3595-3634.

[20] J. Xie, L. Xu, and E. Chen. 2012. Image denoising and inpainting with deep neural networks. In Advances in Neural Information Processing Systems. 341-349.

[21] L. Xu, J. S. Ren, C. Liu, and J. Jia. 2014. Deep Convolutional Neural Network for Image Deconvolution. In Advances in Neural Information Processing Systems 27. Curran Associates, Inc., 1790-1798.

[22] L. Xu, X. Tao, and J. Jia. 2014. Inverse kernels for fast spatial deconvolution. In European Conference on Computer Vision. Springer, 33-48.

[23] M. D. Zeiler and R. Fergus. 2013. Visualizing and understanding convolutional networks. arXiv preprint arXiv:1311.2901 (2013).

[24] K. Zhang, W. Xue, and L. Zhang. 2017. Non-blind image deconvolution using deep dual-pathway rectifier neural network. In IEEE International Conference on Acoustics, Speech and Signal Processing (ICASSP). 2602-2606. 